\title{
(2)

\section{The magnetoelastic problem of a crack in a soft ferromagnetic solid}

\author{
Chun-Bo Lin ${ }^{\text {a }}$, Chau-Shioung Yeh ${ }^{\text {b,* }}$ \\ ${ }^{a}$ Department of Mechanical Engineering, Nan Kai College, Nantou 542, Taiwan, ROC \\ ${ }^{\mathrm{b}}$ Institute of Applied Mechanics, National Taiwan University, Taipei 10764, Taiwan, ROC
}

Received 21 March 2001

\begin{abstract}
The induced magnetoelastic stresses and Maxwell stresses generated by a uniform magnetic field in an infinite soft ferromagnetic medium containing a finite plane crack are analyzed in this paper. The soft ferromagnetic elastic solids with a finite crack are considered to be high magnetic susceptibility materials. By the use of complex variable theory, the exact solutions for magnetic field quantities and both magnetoelastic stresses and Maxwell stresses can be obtained in a closed form. The singularity of stress intensity in the vicinity of crack tip and crack opening condition can also be determined. (c) 2001 Published by Elsevier Science Ltd.
\end{abstract}

Keywords: Magnetoelastic stress; Crack opening condition

\section{Introduction}

Because of the possible application of magnetoelasticity in various fields, such as geophysics, optical acoustics, and many other magnetomechanical devices (Moon, 1984; Maugin, 1988; Eringen and Maugin, 1989), the theory concerning the interaction between the externally applied magnetic field and elastic solid has received rapidly increasing attention in the recent years.

The magnetoelastic interactions in ferromagnetic material was summarized by Brown (1966). On the basis of the large deformation theory of elasticity and classic theory of ferromagnetism, he developed a rigorous phenomenological theory of magnetoelasticity, and Tiersten also derived the consistent set of field equations and boundary conditions by the application of the laws of continuum physics (Tiersten, 1964) and variational principle (Tiersten, 1965). Since their theories are nonlinear and complicated, Pao and Yeh (1973) have developed a linear theory of magnetoelasticity for soft ferromagnetic materials with multidomain structure, which are widely used in magnetomechanical technology. The linear theory has been applied to investigate the buckling behavior of an isotropic plate subjected to a transversally applied magnetic induction (Pao and Yeh, 1973), to find the stress intensity factors of cracks in soft ferromagnetic

\footnotetext{
${ }^{*}$ Corresponding author. Fax: +886-2-363-9290.

E-mail address: csyeh@iam.ntu.edu.tw (C.-S. Yeh).
} 
solids (Shindo, 1977, 1978, 1980), and to calculate the induced magnetic fields in a magnetized elastic halfplane subjected to a mechanical singularity (Yeh, 1989).

In this paper, we investigate the elastic fields induced by the applied magnetic fields on a soft ferromagnetic solid containing a crack, and make study on two-dimension crack problem for the soft ferromagnetic solids subjected to applied uniform magnetostatic fields and far-field stresses. The solution is expressed in a closed form by the use of complex variable technique.

\section{Formulations of magnetic fields}

The static magnetic field satisfies the following equations:

$$
B_{i, i}=0, \quad e_{i j k} H_{k, j}=0
$$

where $B_{i}$ and $H_{k}$ are magnetic induction (or magnetic flux density) and magnetic intensity, respectively; $e_{i j k}$ denotes the permutation symbol and $($,$) is the partial differentiation with respect to the space variable.$ The constitutive law of magnetic field is

$$
B_{i}=\mu_{0}\left(H_{i}+M_{i}\right)=\mu_{0} \mu_{\mathrm{r}} H_{i}
$$

with

$$
M_{i}=\chi H_{i}, \quad \mu_{\mathrm{r}}=1+\chi
$$

where $M_{i}$ is magnetization, $\mu_{0}=4 \pi \times 10^{-7} \mathrm{~N} / \mathrm{A}^{2}$ is a universal constant, $\chi$ and $\mu_{\mathrm{r}}$ are magnetic susceptibility and relative magnetic permeability, respectively. For linear soft ferromagnetic materials, $\chi \sim\left(10^{2} \rightarrow\right.$ $\left.10^{5}\right) \gg 1$ (Moon, 1984).

In view of the second part of Eq. (1), magnetic intensity may be expressed as gradient of a scalar potential $\varphi$ (i.e. $H_{i}=\varphi_{, i}$ ). Furthermore, by the use of the first part of Eqs. (1) and (2), it is concluded that $\varphi$ satisfies Laplace equation (i.e. $\varphi_{, i i}=0$ ). Let us extend to the analytic complex potential function for magnetic fields in terms of complex variable $z=x+\mathrm{i} y$ as

$$
h(z)=\varphi(x, y)+\mathrm{i} \gamma(x, y)
$$

where the real part $\varphi(x, y)$ is a scalar potential function described above and the corresponding function $\gamma(x, y)$ in the imaginary part which also satisfies Laplace equation is derived from Cauchy-Riemann equations. The complex function $h(z)$ relates to the magnetic field quantities as

$$
\begin{aligned}
H_{x}+\mathrm{i} H_{y} & =\frac{\partial \varphi}{\partial x}+\mathrm{i} \frac{\partial \varphi}{\partial y}=\overline{h^{\prime}(z)} \\
B_{x}+\mathrm{i} B_{y} & =\mu_{0} \mu_{\mathrm{r}}\left(\frac{\partial \gamma}{\partial y}-\mathrm{i} \frac{\partial \gamma}{\partial x}\right)=\mu_{0} \mu_{\mathrm{r}} \overline{h^{\prime}(z)}
\end{aligned}
$$

where the complex conjugate of functions is denoted by the overline. The expressions of two magnetic field quantities in terms of $h(z)$ as shown in Eqs. (5) can satisfy field Eq. (1). By integrating along the boundary and the surface of a body, the following two equations can be deduced from Eqs. (1) and (5) as

$$
\begin{aligned}
\int\left(H_{x} \mathrm{~d} x+H_{y} \mathrm{~d} y\right) & =\int\left(\frac{\partial \varphi}{\partial x} \mathrm{~d} x+\frac{\partial \varphi}{\partial y} \mathrm{~d} y\right)=\varphi \\
\int\left(B_{x} n_{x}+B_{y} n_{y}\right) \mathrm{d} S & =\int\left(B_{x} \frac{\mathrm{d} y}{\mathrm{~d} s}-B_{y} \frac{\mathrm{d} x}{\mathrm{~d} s}\right) \mathrm{d} S=\int \mu_{0} \mu_{\mathrm{r}}\left(\frac{\partial \gamma}{\partial y} \mathrm{~d} y+\frac{\partial \gamma}{\partial x} \mathrm{~d} x\right)=\mu_{0} \mu_{\mathrm{r} \gamma}
\end{aligned}
$$

where $\left(n_{x}, n_{y}\right)=(\mathrm{d} y / \mathrm{d} s,-\mathrm{d} x / \mathrm{d} s)$ is the outward unit normal on the surface $S$ of body. Such a definition will keep the body on the left if the path of integral is along counterclockwise direction (England, 1971). It is 
noted that the relation $\mathrm{d} S=1 \mathrm{~d} s$ for unit depth is used in the derivation of Eq. (6). Since the corresponding boundary conditions of Eqs. (1) can be expressed as (Moon, 1984)

$$
\oint_{C}\left(H_{x} \mathrm{~d} x+H_{y} \mathrm{~d} y\right)=0, \quad \int_{S}\left(B_{x} n_{x}+B_{y} n_{y}\right) \mathrm{d} S=0
$$

The area $S$ denotes the surface enclosed by the closed loop $C$. Referring Eqs. (6), the preceding equations are also equivalent to that both $\varphi$ and $\mu_{0} \mu_{\mathrm{r}} \gamma$ are continuous across the boundary. In this section, an infinite soft ferromagnetic material containing a line crack situated along the segment $L=(-a, a)$ of the real axis is shown in Fig. 1. The real axis is represented as the sum of $L$ and $L^{*}$, where $L$ is the region of crack and $L^{*}$ is the rest part. Then following the similar procedure developed by Muskhelishvili (1953) to solve elastic fields around a crack, it is convenient to introduce two complex functions for magnetic fields as

$$
\Phi^{\mathrm{M}}(z)=h^{\prime}(z), \quad \Omega^{\mathrm{M}}(z)=\overline{\Phi^{\mathrm{M}}}(z)
$$

where the superscript $\mathrm{M}$ denotes those quantities related to magnetic fields. The function $\overline{\Phi^{\mathrm{M}}}(z)$ denotes complex conjugate of the coefficients (not argument) in $\Phi^{\mathrm{M}}$. The boundary conditions of magnetic fields along real axis can be expressed as

$$
\begin{aligned}
& \Phi^{\mathrm{M}+}(x)-\Omega^{\mathrm{M}-}(x)=-2 \mathrm{i} \frac{B_{y}^{+}}{\mu_{0} \mu_{\mathrm{r}}} \quad \text { on } L \\
& \Phi^{\mathrm{M}-}(x)-\Omega^{\mathrm{M}+}(x)=-2 \mathrm{i} \frac{B_{y}^{-}}{\mu_{0} \mu_{\mathrm{r}}} \quad \text { on } L
\end{aligned}
$$

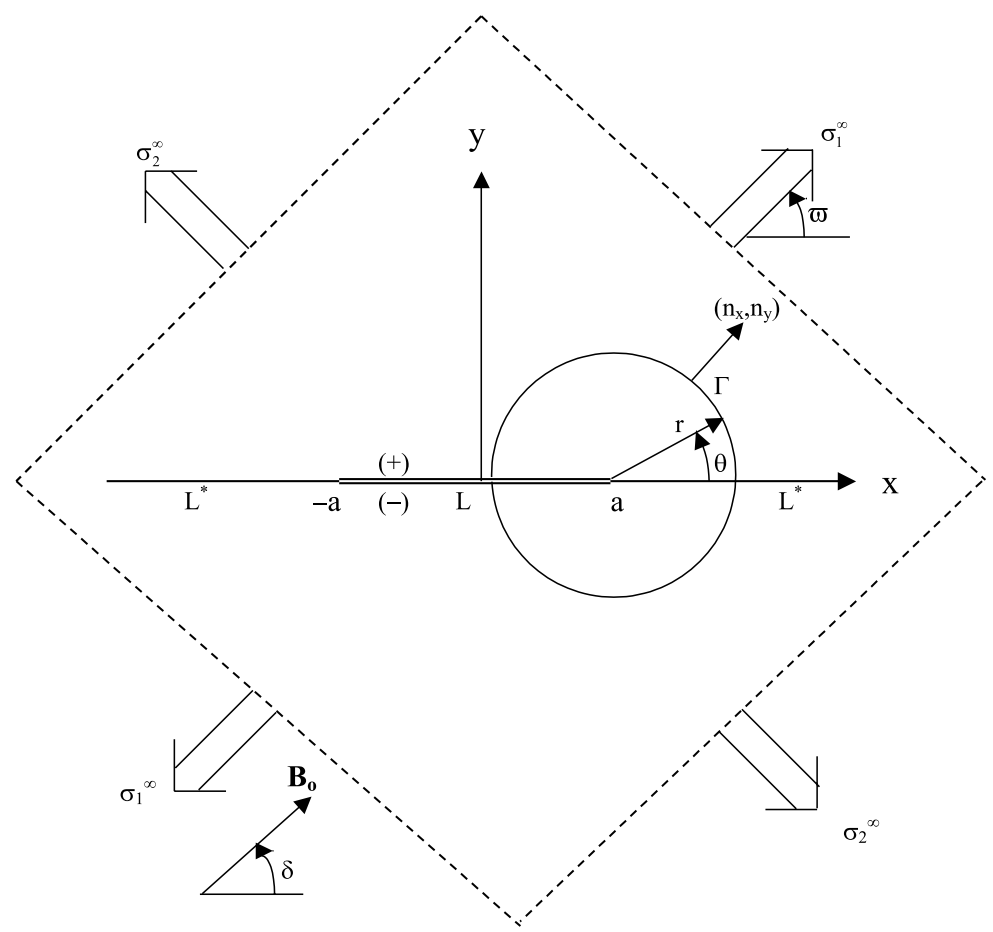

Fig. 1. The far-field stresses and magnetic induction on a soft ferromagnetic solid with a line crack. 
The quantities with superscript + and - denote the upper and lower surfaces of crack as shown in Fig. 1. It should be mentioned that the following equation has been used in the derivation of Eqs. (9) and (10):

$$
\Omega^{\mathrm{M}-}(x)=\lim _{y \rightarrow 0-} \Omega^{\mathrm{M}}(z)=\lim _{y \rightarrow 0+} \Omega^{\mathrm{M}}(\bar{z})=\lim _{y \rightarrow 0+} \overline{\Phi^{\mathrm{M}}(z)}=\overline{\Phi^{\mathrm{M}+}(x)}
$$

Then on adding and subtracting of Eqs. (9) and (10) we have

$$
\begin{aligned}
& {\left[\Phi^{\mathrm{M}}(x)-\Omega^{\mathrm{M}}(x)\right]^{+}+\left[\Phi^{\mathrm{M}}(x)-\Omega^{\mathrm{M}}(x)\right]^{-}=2 \alpha^{\mathrm{M}}(t) \text { on } L} \\
& {\left[\Phi^{\mathrm{M}}(x)+\Omega^{\mathrm{M}}(x)\right]^{+}-\left[\Phi^{\mathrm{M}}(x)+\Omega^{\mathrm{M}}(x)\right]^{-}=2 \beta^{\mathrm{M}}(t) \text { on } L}
\end{aligned}
$$

where $\alpha(t)$ and $\beta(t)$ are related to the magnetic induction on $L$ by

$$
\alpha^{\mathrm{M}}(t)=\frac{-\mathrm{i}}{\mu_{0} \mu_{\mathrm{r}}}\left(B_{y}^{+}+B_{y}^{-}\right), \quad \beta^{\mathrm{M}}(t)=\frac{-\mathrm{i}}{\mu_{0} \mu_{\mathrm{r}}}\left(B_{y}^{+}-B_{y}^{-}\right)
$$

and they must satisfy the Hölder condition on $L$ (England, 1971). Eq. (12) is a Hilbert problem for the function $\Phi(z)-\Omega(z)$ and Eq. (13) is a Plemelj equation for the function $\Phi(z)+\Omega(z)$. Therefore, we can obtain the solutions of both functions as (Muskhelishvili, 1953)

$$
\begin{aligned}
& \Phi^{\mathrm{M}}(z)-\Omega^{\mathrm{M}}(z)=\frac{X(z)}{\pi \mathrm{i}} \int_{L} \frac{\alpha^{\mathrm{M}}(t) \mathrm{d} t}{X^{+}(t)(t-z)}+X(z) P(z) \\
& \Phi^{\mathrm{M}}(z)+\Omega^{\mathrm{M}}(z)=\frac{1}{\pi \mathrm{i}} \int_{L} \frac{\beta^{\mathrm{M}}(t) \mathrm{d} t}{t-z}+Q(z)
\end{aligned}
$$

where the Plemelj function $X(z)$ satisfying the condition $X^{+}(t)=-X^{-}(t)$ may take the form as

$$
X(z)=(z+a)^{-1 / 2}(z-a)^{-1 / 2}
$$

with the selected branch cut such that

$$
\lim _{z \rightarrow \infty}[z X(z)]=1
$$

The singularity in Eq. (17) implies that the near-tip magnetic induction always possesses the characteristic inverse square root singularity in terms of radial distance from the crack tip. Such a feature would not be affected by the discontinuity in magnetic permeability jump across the material interface. The general solution of $\Phi^{\mathrm{M}}$ can be derived from Eqs. (15) and (16)

$$
\Phi^{\mathrm{M}}(z)=\frac{1}{2 \pi \mathrm{i}} \int_{L} \frac{\beta^{\mathrm{M}}(t) \mathrm{d} t}{t-z}+\frac{X(z)}{2 \pi \mathrm{i}} \int_{L} \frac{\alpha^{\mathrm{M}}(t) \mathrm{d} t}{X^{+}(t)(t-z)}+\frac{1}{2} Q(z)+\frac{1}{2} X(z) P(z)
$$

For a single crack problem, the function $P(z)$ can be expressed as a polynomial of degree no more than 1 (Muskhelishvili, 1953), i.e.

$$
P(z)=c_{1} z+c_{0}
$$

Furthermore, the function $Q(z)$ must be a constant since it is holomorphic in the whole material region including small and large $|z|$, i.e.

$$
Q(z)=d
$$

Through the Eqs. (1) and (5), the magnetic intensity $H_{j}$ which is the gradient of potential $\varphi$ plays the role as the driving term of magnetic field and the magnetic induction $B_{j}$ can be viewed as flux term of magnetic field. Therefore, the impedance of magnetic flux can be defined as

$$
Z=\frac{H}{B}=\frac{1}{\mu_{0} \mu_{\mathrm{r}}}
$$


where $H\left(=\left(H_{x}^{2}+H_{y}^{2}\right)^{1 / 2}\right)$ and $B\left(=\left(B_{x}^{2}+B_{y}^{2}\right)^{1 / 2}\right)$ are the strength of magnetic intensity and magnetic induction, respectively. Due to the variation of magnetic permeability between different materials, the applied magnetic induction $B_{0 x}+\mathrm{i} B_{0 y}$ in the soft ferromagnetic body $\left(\mu_{\mathrm{r}} \gg 1\right)$ containing a crack will go around the crack (for air, $\mu_{\mathrm{r}}=1$ ) rather than pass through it. Such a feature is similar to that the magnetic induction in air $\left(\mu_{\mathrm{r}}=1\right)$ will not pass through the superconductor $\left(\mu_{\mathrm{r}}=0\right)$. From the above description, we can treat the boundary of crack as the insulated surface of magnetic induction, i.e.

$$
\alpha^{\mathrm{M}}(t)=\beta^{\mathrm{M}}(t)=0
$$

The solution can be found once the coefficients of $P(z)$ and $Q(z)$ are determined. On the basis of Eqs. (5), (8) and the expression of applied magnetic induction on the soft ferromagnetic material, the functions $\Phi(z)$ and $\Omega(z)$ for large value of $|z|$ take the form

$$
\Phi^{\mathrm{M}}(z)=\Gamma^{\mathrm{M}}+O\left(\frac{1}{z}\right), \quad \Omega^{\mathrm{M}}(z)=\overline{\Gamma^{\mathrm{M}}}+O\left(\frac{1}{z}\right) \quad \text { for }|z| \gg 1
$$

where

$$
\Gamma^{\mathrm{M}}=\frac{1}{\mu_{0} \mu_{\mathrm{r}}}\left(B_{0 x}-\mathrm{i} B_{0 y}\right)
$$

Substituting Eqs. (20) and (21) into Eqs. (15) and (16) and using Eqs. (24), we can obtain

$$
c_{1}=\Gamma^{\mathrm{M}}-\overline{\Gamma^{\mathrm{M}}}, \quad d=\Gamma^{\mathrm{M}}+\overline{\Gamma^{\mathrm{M}}}
$$

By applying Eq. (11), the first part in Eq. (7) can be expressed as

$$
\int_{L}\left[\Phi^{\mathrm{M}+}(t)+\Omega^{\mathrm{M}-}(t)\right] \mathrm{d} t-\int_{L}\left[\Phi^{\mathrm{M}-}(t)+\Omega^{\mathrm{M}+}(t)\right] \mathrm{d} t=0
$$

Thus on the basis of residue theorem and Eqs. (15), (17) and (20), Eq. (27) becomes

$$
\begin{aligned}
\int_{L}\left\{\left[\Phi^{\mathrm{M}}(t)-\Omega^{\mathrm{M}}(t)\right]^{+}-\left[\Phi^{\mathrm{M}}(t)-\Omega^{\mathrm{M}}(t)\right]^{-}\right\} \mathrm{d} t & =\int_{C}\left[\Phi^{\mathrm{M}}(\zeta)-\Omega^{\mathrm{M}}(\zeta)\right] \mathrm{d} \zeta \\
& =\int_{C} \frac{c_{1} \zeta+c_{0}}{\sqrt{(\zeta+a)(\zeta-a)}} \mathrm{d} \zeta=2 \pi \mathrm{i} c_{0}=0
\end{aligned}
$$

where the range of a closed loop $C$ surrounding the crack can be extended from the crack surface to infinity (i.e. $|R| \rightarrow \infty$ ).

Substituting Eqs. (20), (21) and (23) into Eq. (19) and using Eqs. (8), (26) and (28), we have

$$
h^{\prime}(z)=\Phi^{\mathrm{M}}(z)=\frac{1}{2 \mu_{0} \mu_{\mathrm{r}}}\left(\frac{-2 \mathrm{i} B_{0 y} z}{\sqrt{z^{2}-a^{2}}}+2 B_{0 x}\right)=\frac{1}{\mu_{0} \mu_{\mathrm{r}}}\left(B_{0 x}-\mathrm{i} \frac{B_{0 y} z}{\sqrt{z^{2}-a^{2}}}\right)
$$

Then the magnetic field quantities can be obtained from Eqs. (5) and (29) as

$$
H_{x}+\mathrm{i} H_{y}=\frac{1}{\mu_{0} \mu_{\mathrm{r}}}\left(B_{x}+\mathrm{i} B_{y}\right)=\overline{h^{\prime}(z)}=\frac{1}{\mu_{0} \mu_{\mathrm{r}}}\left(B_{0 x}+\mathrm{i} \frac{B_{0 y} \bar{z}}{\sqrt{\bar{z}^{2}-a^{2}}}\right)
$$

\section{Formulations of magnetoelastic fields}

The equation of equilibrium under mechanical and magnetic body forces is (Brown, 1966)

$$
t_{i j, i}+\mu_{0} M_{i} H_{j, i}+F_{j}=\left(t_{i j}+t_{i j}^{\mathrm{M}}\right)_{, i}+F_{j}=0
$$


where $t_{i j}, t_{i j}^{\mathrm{M}}, \mu_{0} M_{i} H_{j, i}$ and $F_{j}$ are the magnetomechanical stress tensor, the Maxwell stress tensor, the magnetic force and the mechanical force per unit volume, respectively. Neglecting the effect of magnetostriction, the constitutive equations are (Pao and Yeh, 1973)

$$
\begin{aligned}
t_{i j} & =\tau_{i j}+\frac{\mu_{0}}{\chi} M_{i} M_{j}, \quad \tau_{i j}=\lambda \delta_{i j} u_{k, k}+G\left(u_{i, j}+u_{j, i}\right) \\
t_{i j}^{\mathrm{M}} & =B_{i} H_{j}-\frac{1}{2} \mu_{0} H_{k} H_{k} \delta_{i j}
\end{aligned}
$$

where $\tau_{i j}$ is the elastic stress tensor for a general nonmagnetizable material. The symbols $\lambda$ and $G$ are Lamé's constants, and $\delta_{i j}$ is Kronecker delta. It is noticed that both the magnetoelastic stress and elastic stress tensor are symmetric for soft ferromagnetic material. Substituting Eq. (32) into Eq. (31) and using Eqs. (1)(3), we have

$$
(\lambda+G) u_{k, k i}+G u_{i, j j}+2 \mu_{0} \chi H_{j} H_{i, j}+F_{i}=0
$$

or in complex form

$$
2(\lambda+G) \frac{\partial}{\partial \bar{z}}\left(\frac{\partial D}{\partial z}+\frac{\overline{\partial D}}{\partial z}\right)+4 G \frac{\partial^{2} D}{\partial z \partial \bar{z}}+2 \mu_{0} \chi\left[\overline{h^{\prime}(z)} \frac{\partial}{\partial z}+h^{\prime}(z) \frac{\partial}{\partial \bar{z}}\right] \overline{h^{\prime}(z)}+\frac{\partial V}{\partial \bar{z}}=0
$$

with

$$
D=u_{x}+\mathrm{i} u_{y}, \quad F_{x}+\mathrm{i} F_{y}=\frac{\partial V}{\partial x}=\frac{\partial V}{\partial z}=\frac{\partial V}{\partial \bar{z}}
$$

where $D$ is complex displacement and $V(z)$ is force potential which is real (England, 1971). Since $h(z)$ is an analytic function, we can obtain

$$
\frac{\partial}{\partial z} \overline{h^{\prime}(z)}=\frac{\partial^{2} \overline{h(z)}}{\partial z \partial \bar{z}}=0
$$

Then Eq. (34) takes the form

$$
\frac{\partial}{\partial \bar{z}} \vartheta^{\prime}=0
$$

where

$$
\vartheta^{\prime}(z)=2(\lambda+G)\left(\frac{\partial D}{\partial z}+\frac{\overline{\partial D}}{\partial z}\right)+4 G \frac{\partial D}{\partial z}+2 \mu_{0} \chi h^{\prime}(z) \overline{h^{\prime}(z)}+V(z)
$$

The real and imaginary parts of $\partial D / \partial z$ can be calculated by taking $\vartheta^{\prime}$ and its complex conjugate. Hence

$$
\begin{aligned}
& 4(\lambda+2 G) \operatorname{Re}\left(\frac{\partial D}{\partial z}\right)+2 \mu_{0} \chi h^{\prime}(z) \overline{h^{\prime}(z)}=-V(z)+\frac{1}{2}\left(\vartheta^{\prime}(z)+\overline{\vartheta^{\prime}(z)}\right) \\
& 4 G \operatorname{Im}\left(\frac{\partial D}{\partial z}\right)=\frac{1}{2 \mathrm{i}}\left(\vartheta^{\prime}(z)-\overline{\vartheta^{\prime}(z)}\right)
\end{aligned}
$$

and then

$$
8 G(\lambda+2 G) \frac{\partial D}{\partial z}+4 G \mu_{0} \chi h^{\prime}(z) \overline{h^{\prime}(z)}=(\lambda+3 G) \vartheta^{\prime}(z)-(\lambda+G) \overline{\vartheta^{\prime}(z)}-2 G V(z)
$$

The integration of Eq. (40) with respect to $z$ yields

$$
8 G(\lambda+2 G) D=(\lambda+3 G) \vartheta(z)-(\lambda+G) \overline{\vartheta^{\prime}(z)}-4 G \mu_{0} \chi \int h^{\prime}(z) \overline{h^{\prime}(z)} \mathrm{d} z+\overline{\omega(z)}-2 G Y(z)
$$


or

$$
2 G D=\kappa \phi(z)-z \overline{\phi^{\prime}(z)}-\overline{\psi(z)}-\frac{G}{(\lambda+2 G)} \mu_{0} \chi h(z) \overline{h^{\prime}(z)}-\frac{G}{2(\lambda+2 G)} Y(z)
$$

where

$$
\phi(z)=\frac{\lambda+G}{4(\lambda+2 G)} \vartheta(z), \quad \psi(z)=-\frac{1}{4(\lambda+2 G)} \omega(z), \quad \kappa=\frac{\lambda+3 G}{\lambda+G}, \quad Y(z)=\int V(z) \mathrm{d} z
$$

The symbol $\overline{\omega(z)}$ is an arbitrary function.

The stress components in Eqs. (32) can be expressed in terms of the complex displacement and magnetic field as

$$
\begin{aligned}
\left(t_{x x}+t_{y y}\right)^{\mathrm{T}} & =\left(t_{x x}+t_{y y}\right)+\left(t_{x x}+t_{y y}\right)^{\mathrm{M}} \\
\left(t_{x x}-t_{y y}+2 \mathrm{i} t_{x y}\right)^{\mathrm{T}} & =\left(t_{x x}-t_{y y}+2 \mathrm{i} t_{x y}\right)+\left(t_{x x}-t_{y y}+2 \mathrm{i} t_{x y}\right)^{\mathrm{M}}
\end{aligned}
$$

and

$$
\left(t_{y y}-\mathrm{i} t_{x y}\right)^{\mathrm{T}}=\left(t_{y y}-\mathrm{i} t_{x y}\right)+\left(t_{y y}-\mathrm{i} t_{x y}\right)^{\mathrm{M}}
$$

where

$$
\begin{aligned}
& t_{x x}+t_{y y}=2(\lambda+G)\left(\frac{\partial D}{\partial z}+\frac{\overline{\partial D}}{\partial z}\right)+\frac{\mu_{0}}{\chi}\left(M_{x}^{2}+M_{y}^{2}\right)=2\left[\phi^{\prime}(z)+\overline{\phi^{\prime}(z)}\right]+\mu_{0} \chi h^{\prime}(z) \overline{h^{\prime}(z)}-\frac{\lambda+G}{\lambda+2 G} V(z) \\
& \left(t_{x x}+t_{y y}\right)^{\mathrm{M}}=B_{x} H_{x}+B_{y} H_{y}-\mu_{0}\left(H_{x}^{2}+H_{y}^{2}\right)=\mu_{0} \chi h^{\prime}(z) \overline{h^{\prime}(z)} \\
& t_{x x}-t_{y y}+2 \mathrm{i} t_{x y}=4 G \frac{\partial D}{\partial \bar{z}}+\frac{\mu_{0}}{\chi}\left(M_{x}^{2}-M_{y}^{2}+2 \mathrm{i} M_{x} M_{y}\right)=-2\left[z \overline{\phi^{\prime \prime}(z)}+\overline{\psi^{\prime}(z)}\right]+\mu_{0} \chi \overline{h^{\prime}(z)} \overline{h^{\prime}(z)}-\frac{\lambda+G}{\lambda+2 G} \frac{\partial Y}{\partial \bar{z}} \\
& \left(t_{x x}-t_{y y}+2 \mathrm{i} t_{x y}\right)^{\mathrm{M}}=B_{x} H_{x}-B_{y} H_{y}+2 \mathrm{i} B_{x} H_{y}=\mu_{0} \mu_{\mathrm{r}} \overline{h^{\prime}(z)} \overline{h^{\prime}(z)} \\
& t_{y y}-\mathrm{i} t_{x y}=\phi^{\prime}(z)+\overline{\phi^{\prime}(z)}+\left[z \overline{\phi^{\prime \prime}(z)}+\overline{\psi^{\prime}(z)}\right]+\frac{1}{2} \mu_{0} \chi\left[h^{\prime}(z) \overline{h^{\prime}(z)}-\overline{h^{\prime}(z)} \overline{h^{\prime}(z)}\right]-\frac{1}{2} \frac{\lambda+G}{\lambda+2 G}\left[V(z)-\frac{\partial Y}{\partial \bar{z}}\right] \\
& \left(t_{y y}-\mathrm{i} t_{x y}\right)^{\mathrm{M}}=\frac{1}{2} \mu_{0}\left[\chi h^{\prime}(z) \overline{h^{\prime}(z)}-\mu_{\mathrm{r}} \overline{h^{\prime}(z)} \overline{h^{\prime}(z)}\right]
\end{aligned}
$$

and the stresses with superscript $\mathrm{T}$ denote the total stresses which are the sum of magnetoelastic stresses and Maxwell stresses. Since the Maxwell stresses are not related to the deformation and are defined artificially just for the sake of convenience, only the magnetoelastic stresses rather than the total stresses are practical for the present problem. Thereafter, the surface traction on boundary of a body can be expressed by stress components in a complex form as (England, 1971)

$$
P+\mathrm{i} Q=\left(t_{x x}+\mathrm{i} t_{x y}\right)^{\mathrm{T}} \frac{\mathrm{d} y}{\mathrm{~d} s}-\mathrm{i}\left(t_{y y}-\mathrm{i} t_{x y}\right)^{\mathrm{T}} \frac{\mathrm{d} x}{\mathrm{~d} s}=\frac{-\mathrm{i}}{2}\left[\left(t_{x x}+t_{y y}\right)^{\mathrm{T}} \frac{\mathrm{d} z}{\mathrm{~d} s}-\left(t_{x x}-t_{y y}+2 \mathrm{i} t_{x y}\right)^{\mathrm{T}} \frac{\mathrm{d} \bar{z}}{\mathrm{~d} s}\right]
$$

Hence by the use of Eqs. (46) and (47), the resultant force $R(z)$ over the arbitrary arc $A B$ on the boundary of the body is obtained 


$$
\begin{aligned}
R(z)= & \int_{A}^{B}(P+\mathrm{i} Q) \mathrm{d} s \\
= & -\left.\mathrm{i}\left[\phi(z)+z \overline{\phi^{\prime}(z)}+\overline{\psi(z)}+\mu_{0} \chi h(z) \overline{h^{\prime}(z)}-\frac{\mu_{0}}{2}\left(\mu_{\mathrm{r}}+\chi\right) \overline{h(z)} \overline{h^{\prime}(z)}\right]\right|_{A} ^{B} \\
& +\mathrm{i} \mu_{0} \int_{A}^{B} \overline{h^{\prime \prime}(z)}\left[\chi h(z)-\frac{1}{2}\left(\mu_{\mathrm{r}}+\chi\right) \overline{h(z)}\right] \mathrm{d} \bar{z}
\end{aligned}
$$

On the basis of Eqs. (42) and (48), the solutions of mechanical field can be determined such that the displacement boundary condition defined in part of boundary and traction boundary condition defined in the rest part of boundary, respectively, are satisfied.

\section{Magnetoelastic fields around a crack}

Following the similar procedure for determining elastic fields around a crack by Muskhelishvili (1953) and neglecting the body force, we introduce two complex functions for magnetoelastic fields as

$$
\begin{aligned}
& \Phi(z)=\phi^{\prime}(z) \\
& \Omega(z)=\bar{\Phi}(z)+z \overline{\Phi^{\prime}}(z)+\overline{\psi^{\prime}}(z)-\frac{\mu_{0}}{2} \bar{h}^{\prime}(z) \bar{h}^{\prime}(z)
\end{aligned}
$$

The stress components $\left(t_{y y}-\mathrm{i} t_{x y}\right)^{\mathrm{T}}$ in Eq. (45) can be expressed in terms of $\Phi(z)$ and $\Omega(z)$ as

$$
\left(t_{y y}-\mathrm{i} t_{x y}\right)^{\mathrm{T}}=\Phi(z)+\Omega(\bar{z})+(z-\bar{z}) \Phi^{\prime}(z)+\mu_{0} \chi\left[h^{\prime}(z)-\overline{h^{\prime}(z)}\right] \overline{h^{\prime}(z)}
$$

Therefore, using the feature that the function $h^{\prime}(z)$ in Eq. (29) is real on the crack $L$, the traction boundary conditions in the upper and lower surfaces of crack are expressed as

$$
\begin{aligned}
& \Phi^{+}(x)+\Omega^{-}(x)=\left(t_{y y}-\mathrm{i} t_{x y}\right)^{\mathrm{T}+}=P^{+} \quad \text { on } L \\
& \Phi^{-}(x)+\Omega^{+}(x)=\left(t_{y y}-\mathrm{i} t_{x y}\right)^{\mathrm{T}-}=P^{-} \text {on } L
\end{aligned}
$$

Then adding and subtracting of Eqs. (51) and (52) we have

$$
\begin{aligned}
& {[\Phi(x)+\Omega(x)]^{+}+[\Phi(x)+\Omega(x)]^{-}=2 \alpha(t) \text { on } L} \\
& {[\Phi(x)-\Omega(x)]^{+}-[\Phi(x)-\Omega(x)]^{-}=2 \beta(t) \text { on } L}
\end{aligned}
$$

where

$$
\alpha(t)=\frac{1}{2}\left(P^{+}+P^{-}\right), \quad \beta(t)=\frac{1}{2}\left(P^{+}-P^{-}\right)
$$

must satisfy the Hölder condition on $L$. The solutions of the Eqs. (53) and (54) are

$$
\begin{aligned}
& \Phi(z)+\Omega(z)=\frac{X(z)}{\pi \mathrm{i}} \int_{L} \frac{\alpha(t) \mathrm{d} t}{X^{+}(t)(t-z)}+X(z) T(z) \\
& \Phi(z)-\Omega(z)=\frac{1}{\pi \mathrm{i}} \int_{L} \frac{\beta(t) \mathrm{d} t}{t-z}+U(z)
\end{aligned}
$$

where the Plemelj function has been defined in Eq. (17) with branch cut shown in Eq. (18). Therefore, the general solution of $\Phi$ can be obtained as 


$$
\begin{aligned}
& \Phi(z)=\frac{X(z)}{2 \pi \mathrm{i}} \int_{L} \frac{\alpha(t) \mathrm{d} t}{X^{+}(t)(t-z)}+\frac{1}{2 \pi \mathrm{i}} \int_{L} \frac{\beta(t) \mathrm{d} t}{t-z}+\frac{1}{2} X(z) T(z)+\frac{1}{2} U(z) \\
& \Omega(z)=\frac{X(z)}{2 \pi \mathrm{i}} \int_{L} \frac{\alpha(t) \mathrm{d} t}{X^{+}(t)(t-z)}-\frac{1}{2 \pi \mathrm{i}} \int_{L} \frac{\beta(t) \mathrm{d} t}{t-z}+\frac{1}{2} X(z) T(z)-\frac{1}{2} U(z)
\end{aligned}
$$

For a single crack with surface free of traction, we can obtain

$$
\alpha(t)=\beta(t)=P^{+}=P^{-}=0
$$

and the function $R(z)$ can be expressed as a polynomial of degree no more than 1 (Muskhelishvili, 1953), i.e.

$$
T(z)=e_{1} z+e_{0}
$$

Furthermore, analogous to the derivation of Eq. (21) for magnetic field, the function $U(z)$ is a constant since it is holomorphic for small and large $|z|$, i.e.

$$
U(z)=f
$$

In order to determine the coefficients $e_{1}, e_{0}$ and $f$, it is necessary to consider the conditions at infinity. Let us assume that

$$
\phi^{\prime}(z)=(A+\mathrm{i} R)+O\left(\frac{1}{z}\right), \quad \psi^{\prime}(z)=(J+\mathrm{i} K)+O\left(\frac{1}{z}\right) \quad \text { for }|z| \gg 1
$$

The symbols $A, R, J$ and $K$ are constants which can be determined from the magnetoelastic stress conditions at infinity. It is convenient to consider that the magnetic induction is generated at outside medium (such as air) and applied normally across the edge of soft ferromagnetic material as shown in Fig. 1. In view of Eqs. (31) and (47), the total stresses rather than the magnetoelastic stresses on the boundary surface are continuous in the absence of body force. Substituting Eqs. (24) and (62) into Eq. (44) and neglecting body force, we have

$$
\begin{gathered}
\sigma_{1}^{\infty}+\sigma_{2}^{\infty}+\frac{1}{2 \mu_{0}}\left(B_{0 x}^{2}+B_{0 y}^{2}\right)=4 A+\frac{2 \chi}{\mu_{0} \mu_{\mathrm{r}}^{2}}\left(B_{0 x}^{2}+B_{0 y}^{2}\right) \\
\left(\sigma_{1}^{\infty}-\sigma_{2}^{\infty}\right) \mathrm{e}^{2 \mathrm{i} \varpi}+\frac{1}{2 \mu_{0}}\left(B_{0 x}^{2}+B_{0 y}^{2}\right) \mathrm{e}^{2 \mathrm{i} \delta}=-2(J-\mathrm{i} K)+\frac{\left(\mu_{\mathrm{r}}+\chi\right)}{\mu_{0} \mu_{\mathrm{r}}^{2}}\left(B_{0 x}^{2}+2 \mathrm{i} B_{0 x} B_{0 y}-B_{0 y}^{2}\right)
\end{gathered}
$$

to obtain the constants $A, J$ and $K$, in which $\left(\sigma_{1}^{\infty}+\mathrm{i} \sigma_{2}^{\infty}\right)$ are the principal stresses at infinity. The symbol $\varpi$ denotes the angle between $\sigma_{1}^{\infty}$ and the real (or $\left.x\right)$ axis and $\delta \equiv \tan ^{-1}\left(B_{0 y} / B_{0 x}\right)$ denotes the angle between the applied magnetic induction and the real axis as depicted in Fig. 1. Aside from the applied stresses, the Maxwell stress $\left(B_{0 x}^{2}+B_{0 y}^{2}\right) / 2 \mu_{0}$ is generated by the applied magnetic induction. The discontinuity of Maxwell stresses across the material boundary was also mentioned by Pao and Yeh (1973) and Moon (1984). The relation between the quantity $C$ and the rotation at infinity $\omega^{\infty}$ can be written as

$$
\omega^{\infty}=\operatorname{Im}(\partial D / \partial z)=(1+\kappa) R /(2 G)
$$

The substitution of Eqs. (63) and (64) into Eq. (62) together with the relation $\left(B_{0 x}^{2}+B_{0 y}^{2}\right) \mathrm{e}^{2 \mathrm{i} \delta}=\left(B_{0 x}^{2}+\right.$ $\left.2 \mathrm{i} B_{0 x} B_{0 y}-B_{0 y}^{2}\right)$ derived from $\delta=\tan ^{-1}\left(B_{0 y} / B_{0 x}\right)$ gives

$$
\begin{aligned}
& \phi^{\prime}(z)=\Gamma+\frac{1}{2 \mu_{0}}\left(\frac{1}{4}-\frac{\chi}{\mu_{\mathrm{r}}^{2}}\right)\left(B_{0 x}^{2}+B_{0 y}^{2}\right)+O\left(\frac{1}{z}\right) \\
& \psi^{\prime}(z)=\Gamma^{\prime}-\frac{1}{2 \mu_{0}}\left(\frac{1}{2}-\frac{\mu_{\mathrm{r}}+\chi}{\mu_{\mathrm{r}}^{2}}\right)\left(B_{0 x}^{2}-2 \mathrm{i} B_{0 x} B_{0 y}-B_{0 y}^{2}\right)+O\left(\frac{1}{z}\right) \text { for }|z| \gg 1
\end{aligned}
$$

where 


$$
\Gamma=\frac{1}{4}\left(\sigma_{1}^{\infty}+\sigma_{2}^{\infty}\right)+\mathrm{i} \frac{2 G \omega^{\infty}}{1+\kappa}, \quad \Gamma^{\prime}=-\frac{1}{2}\left(\sigma_{1}^{\infty}-\sigma_{2}^{\infty}\right) \mathrm{e}^{-2 \mathrm{i} \pi}
$$

Note that the functions $\Phi(z)$ and $\Omega(z)$ must satisfy the conditions at infinity, and then can be expressed as

$$
\begin{aligned}
& \Phi(z)=\Gamma+\frac{1}{2 \mu_{0}}\left(\frac{1}{4}-\frac{\chi}{\mu_{\mathrm{r}}^{2}}\right)\left(B_{0 x}^{2}+B_{0 y}^{2}\right)+O\left(\frac{1}{z}\right) \\
& \Omega(z)=\bar{\Gamma}+\overline{\Gamma^{\prime}}-\frac{1}{2 \mu_{0}}\left[\left(\frac{1}{4}-\frac{\chi}{\mu_{\mathrm{r}}^{2}}\right)\left(B_{0 x}^{2}+4 \mathrm{i} B_{0 x} B_{0 y}-3 B_{0 y}^{2}\right)\right]+O\left(\frac{1}{z}\right) \text { for }|z| \gg 1
\end{aligned}
$$

Substituting Eqs. (60) and (61) into Eqs. (56) and (57) and using Eq. (67), we have

$$
\begin{aligned}
& e_{1}=\Gamma+\bar{\Gamma}+\overline{\Gamma^{\prime}}+\frac{1}{2 \mu_{0}}\left(1-\frac{4 \chi}{\mu_{\mathrm{r}}^{2}}\right)\left(B_{0 y}^{2}-\mathrm{i} B_{0 x} B_{0 y}\right) \\
& f=\Gamma-\bar{\Gamma}-\overline{\Gamma^{\prime}}+\frac{1}{4 \mu_{0}}\left(1-\frac{4 \chi}{\mu_{\mathrm{r}}^{2}}\right)\left(B_{0 x}^{2}+2 \mathrm{i} B_{0 x} B_{0 y}-B_{0 y}^{2}\right)
\end{aligned}
$$

The remaining constant $e_{0}$ can be determined from the condition that the displacement must be single valued, i.e. the displacement must revert to its original value when $z$ describes the point on the contour $C$ which encloses the crack. The derivative of displacement along upper surface of the crack can be deduced from Eqs. (42) and (49) by dropping the body force,

$$
\begin{aligned}
\left(\frac{\partial D}{\partial t}\right)^{+} & =\left[u_{x}^{\prime}(t)+\mathrm{i} u_{y}^{\prime}(t)\right]^{+} \\
& =\frac{1}{2 G}\left\{\left[\kappa \Phi^{+}(t)-\Omega^{-}(t)-\frac{\mu_{0}}{2} \bar{h}^{\prime^{-}}(t) \bar{h}^{\prime^{-}}(t)-\frac{G}{\lambda+2 G} \mu_{0} \chi h^{\prime^{+}}(t) \bar{h}^{\prime^{+}}(t)\right]\right\} \quad \text { for } t \in L
\end{aligned}
$$

The integration of displacement derivative around the crack surface will become zero such that the singlevalued displacement can be assured, i.e.

$$
\begin{aligned}
\frac{1}{2 G}\{ & \int_{L}\left[\kappa \Phi^{+}(t)-\Omega^{-}(t)-\frac{\mu_{0}}{2} \overline{h^{\prime}}(t) \overline{h^{\prime}}(t)-\frac{G}{\lambda+2 G} \mu_{0} \chi h^{\prime^{+}}(t) \overline{h^{+}}(t)\right] \mathrm{d} t \\
& \left.-\int_{L}\left[\kappa \Phi^{-}(t)-\Omega^{+}(t)-\frac{\mu_{0}}{2} \overline{h^{+}}(t) \overline{h^{\prime^{+}}}(t)-\frac{G}{\lambda+2 G} \mu_{0} \chi h^{\prime^{-}}(t) \overline{h^{\prime-}}(t)\right] \mathrm{d} t\right\}=0
\end{aligned}
$$

This equation can be rearranged as

$$
\begin{aligned}
& \int_{L}\left\{\kappa\left[\Phi^{+}(t)-\Phi^{-}(t)\right]+\left[\Omega^{+}(t)-\Omega^{-}(t)\right]+\frac{\mu_{0}}{2}\left[\bar{h}^{\prime^{+}}(t) \bar{h}^{\prime^{+}}(t)-{\overline{h^{\prime}}}^{-}(t){\overline{h^{\prime}}}^{-}(t)\right]\right. \\
& \left.-\frac{G}{(\lambda+2 G)} \mu_{0} \chi\left[h^{\prime^{+}}(t) \bar{h}^{\prime^{+}}(t)-{h^{\prime}}^{\prime^{-}}(t) \bar{h}^{\prime^{-}}(t)\right]\right\} \mathrm{d} t \\
& =\int_{C}\left[\kappa \Phi(\zeta)+\Omega(\zeta)+\frac{\mu_{0}}{2} \bar{h}^{\prime}(\zeta) \bar{h}^{\prime}(\zeta)-\frac{G}{(\lambda+2 G)} \mu_{0} \chi h^{\prime}(\zeta) \bar{h}^{\prime}(\zeta)\right] \mathrm{d} \zeta=0
\end{aligned}
$$

where the closed loop $C$ surrounding the crack can be extended from the crack surface to infinity (i.e. $|R| \rightarrow \infty)$. The substitution of Eq. (58) into Eq. (71) with using Eqs. (17), (29) and (59)-(61) yields

$$
e_{0}=0
$$


Then substituting Eqs. (59)-(61) into Eq. (58) and using Eqs. (66), (68) and (72), we obtain

$$
\begin{aligned}
\phi^{\prime}(z)= & \Phi(z) \\
= & \frac{z}{4 \sqrt{z^{2}-a^{2}}}\left[\left(\sigma_{1}^{\infty}+\sigma_{2}^{\infty}\right)-\left(\sigma_{1}^{\infty}-\sigma_{2}^{\infty}\right) \mathrm{e}^{2 \mathrm{i} \varpi}\right]+\left[\mathrm{i} \frac{2 G \omega^{\infty}}{1+\kappa}+\frac{1}{4}\left(\sigma_{1}^{\infty}-\sigma_{2}^{\infty}\right) \mathrm{e}^{2 \mathrm{i} \varpi}\right] \\
& -\frac{1}{\mu_{0}}\left(\frac{1}{4}-\frac{\chi}{\mu_{\mathrm{r}}^{2}}\right)\left(B_{0 x}+\mathrm{i} B_{0 y}\right)\left[\frac{\mathrm{i} B_{0 y} z}{\sqrt{z^{2}-a^{2}}}-\frac{1}{2}\left(B_{0 x}+\mathrm{i} B_{0 y}\right)\right]
\end{aligned}
$$

By making use of Eqs. (46), (50), (58) and (73), the magnetoelastic stresses can be expressed in an explicit form as

$$
\begin{aligned}
t_{y y}-\mathrm{i} t_{x y}=\frac{e_{1}}{2} & \left(\frac{z}{\sqrt{z^{2}-a^{2}}}+\frac{\bar{z}}{\sqrt{\bar{z}^{2}-a^{2}}}\right)+\frac{\bar{e}_{1}}{2} \frac{(\bar{z}-z) a^{2}}{\left(\bar{z}^{2}-a^{2}\right)^{3 / 2}}+\frac{1}{2} \frac{1}{\mu_{0} \mu_{\mathrm{r}}^{2}}\left\{B_{0 x}^{2}+\mathrm{i} B_{0 x} B_{0 y}\right. \\
\times & {\left.\left[\frac{(2-\chi) \bar{z}}{\sqrt{\bar{z}^{2}-a^{2}}}-\frac{\chi z}{\sqrt{z^{2}-a^{2}}}\right]+B_{0 y}^{2} \frac{\bar{z}}{\sqrt{\bar{z}^{2}-a^{2}}}\left[\frac{\chi z}{\sqrt{z^{2}-a^{2}}}-\frac{(1-\chi) \bar{z}}{\sqrt{\bar{z}^{2}-a^{2}}}\right]\right\} }
\end{aligned}
$$

where

$$
\begin{aligned}
e_{1} & =e_{1 \mathrm{R}}+\mathrm{i} e_{1 \mathrm{I}} \\
& =\sigma_{1}^{\infty} \sin ^{2} \varpi+\sigma_{2}^{\infty} \cos ^{2} \varpi+\frac{1}{2 \mu_{0}}\left(1-\frac{4 \chi}{\mu_{\mathrm{r}}^{2}}\right) B_{0 y}^{2}-\mathrm{i}\left[\left(\sigma_{1}^{\infty}-\sigma_{2}^{\infty}\right) \sin \varpi \cos \varpi+\frac{1}{2 \mu_{0}}\left(1-\frac{4 \chi}{\mu_{\mathrm{r}}^{2}}\right) B_{0 x} B_{0 y}\right] \\
& \approx \sigma_{1}^{\infty} \sin ^{2} \varpi+\sigma_{2}^{\infty} \cos ^{2} \varpi+\frac{B_{0 y}^{2}}{2 \mu_{0}}-\mathrm{i}\left[\left(\sigma_{1}^{\infty}-\sigma_{2}^{\infty}\right) \sin \varpi \cos \varpi+\frac{B_{0 x} B_{0 y}}{2 \mu_{0}}\right]
\end{aligned}
$$

is rearranged from Eq. (68), and $e_{\mathrm{IR}}$ and $e_{\mathrm{II}}$ are taken as real and imaginary part, respectively. The term $1-4 \chi / \mu_{\mathrm{r}}^{2} \approx 1$ for linear soft ferromagnetic material with $\chi$ and $\mu_{\mathrm{r}}(=\chi+1) \gg 1$ can guarantee the last approximation in Eq. (75). The stress intensity factor of Maxwell stresses near the crack tip $\left(k_{\mathrm{I}}-\mathrm{i} k_{\mathrm{II}}\right)^{\mathrm{M}}$ can be derived from Eqs. (30) and (46) and defined as

$$
\left(k_{\mathrm{I}}-\mathrm{i} k_{\mathrm{II}}\right)^{\mathrm{M}}=\lim _{z \rightarrow a} 2(z-a)\left(t_{y y}-\mathrm{i} t_{x y}\right)^{\mathrm{M}}=\frac{1}{\mu_{0} \mu_{\mathrm{r}}^{2}} \frac{2 \chi+1}{2} a B_{0 y}^{2}
$$

Then the Maxwell stresses at a point near the crack tip (i.e. $r \ll 1$ ) can be expressed in a form

$$
\begin{aligned}
t_{x x}^{\mathrm{M}} & =\frac{k_{\mathrm{I}}^{\mathrm{M}}}{2 r}\left(\sin ^{2} \frac{\theta}{2}-\frac{1}{2 \chi+1} \cos ^{2} \frac{\theta}{2}\right), \quad t_{y y}^{\mathrm{M}}=\frac{k_{\mathrm{I}}^{\mathrm{M}}}{2 r}\left(\cos ^{2} \frac{\theta}{2}-\frac{1}{2 \chi+1} \sin ^{2} \frac{\theta}{2}\right), \\
t_{x y}^{\mathrm{M}} & =\frac{k_{\mathrm{I}}^{\mathrm{M}}}{2 r}\left[\frac{2(\chi+1)}{2 \chi+1} \sin \frac{\theta}{2} \cos \frac{\theta}{2}\right]
\end{aligned}
$$

where the polar coordinates $r$ and $\theta$ as shown in Fig. 1 take the crack tip $(a, 0)$ as origin and relate to $z$ as $z=a+r \mathrm{e}^{\mathrm{i} \theta}$. By the use of Eqs. (29), (46), (50) and (73), the stress intensity factor of magnetoelastic stresses can be defined as function of the distance measured from the crack tip. In view of Eqs. (32), (46) and (74), it is noticed that those terms in Eq. (74) related to $e_{1}$ come from $\tau_{i j}$ with singularity $1 / \sqrt{r}$ near the crack tip and the others come from $\mu_{0} M_{i} M_{j} / \chi$ with singularity $1 / r$ near the crack tip just like the Maxwell stresses. Since these two parts attenuating with the distance $r$ measured from crack tip along positive real axis are different, we investigate the singularity of stress intensity factor for three ranges:

Range 1: $200 / \mu_{r}^{2}<r / a$

In this range, the terms with singularity $1 / \sqrt{r}$ (i.e. $\tau_{i j}$ ) are dominant. Then the stress intensity factor for the mode I, $k_{\mathrm{I}}$ and mode II, $k_{\mathrm{II}}$ of magnetoelastic stresses at point $z$ along positive real axis outside the crack can be expressed as 


$$
\left(k_{\mathrm{I}}-\mathrm{i} k_{\mathrm{II}}\right)=\lim _{z \rightarrow a} \sqrt{2(z-a)}\left(t_{y y}-\mathrm{i} t_{x y}\right) \approx \lim _{z \rightarrow a} 2 \sqrt{2(z-a)} \Phi(z)=\sqrt{a}\left(e_{1}+\mathrm{i} \frac{1-\chi}{2 \mu_{0} \mu_{\mathrm{r}}^{2}} B_{0 x} B_{0 y}\right) \approx \sqrt{a} e_{1}
$$

where the relation $(1-\chi) / \mu_{\mathrm{r}}^{2} \ll 1$ has been used in the absence of mechanical loads $\sigma_{1}^{\infty}$ and $\sigma_{2}^{\infty}$ to assure $(1-\chi) B_{0 x} B_{0 y} / 2 \mu_{0} \mu_{\mathrm{r}}^{2} \ll B_{0 x} B_{0 y} / 2 \mu_{0} \approx e_{1 \mathrm{II}}$. The corresponding magnetoelastic stresses of the point near the crack tip can be expressed as

$$
\begin{aligned}
t_{x x} & =\frac{k_{\mathrm{I}}}{\sqrt{2 r}} \cos \frac{\theta}{2}\left(1-\sin \frac{\theta}{2} \sin \frac{3 \theta}{2}\right)-\frac{k_{\mathrm{II}}}{\sqrt{2 r}} \sin \frac{\theta}{2}\left(2+\cos \frac{\theta}{2} \cos \frac{3 \theta}{2}\right) \\
t_{y y} & =\frac{k_{\mathrm{I}}}{\sqrt{2 r}} \cos \frac{\theta}{2}\left(1+\sin \frac{\theta}{2} \sin \frac{3 \theta}{2}\right)+\frac{k_{\mathrm{II}}}{\sqrt{2 r}} \sin \frac{\theta}{2} \cos \frac{\theta}{2} \cos \frac{3 \theta}{2} \\
t_{x y} & =\frac{k_{\mathrm{I}}}{\sqrt{2 r}} \sin \frac{\theta}{2} \cos \frac{\theta}{2} \cos \frac{3 \theta}{2}+\frac{k_{\mathrm{II}}}{\sqrt{2 r}} \cos \frac{\theta}{2}\left(1-\sin \frac{\theta}{2} \sin \frac{3 \theta}{2}\right)
\end{aligned}
$$

Range 2: $1 /\left(50 \mu_{r}^{2}\right) \leqslant r / a \leqslant 200 / \mu_{r}^{2}$

For the intermediate range of $r$, both terms with singularity $1 / \sqrt{r}$ (i.e. $\tau_{i j}$ ) and $1 / r$ (i.e. $\mu_{0} M_{i} M_{j} / \chi$ ) are comparable to each other, the stress intensity factor of magnetoelastic stresses $k_{\mathrm{I}}-\mathrm{i} k_{\mathrm{II}}$ reduces to

$$
\begin{aligned}
\left(k_{\mathrm{I}}-\mathrm{i} k_{\mathrm{II}}\right) & =\lim _{z \rightarrow a} \sqrt{2(z-a)}\left(t_{y y}-\mathrm{i} t_{x y}\right)=\lim _{z \rightarrow a} \sqrt{2(z-a)}\left[2 \Phi(z)+\frac{2 \chi-1}{2} \mu_{0} h^{\prime}(z) \overline{h^{\prime}(z)}\right] \\
& =\sqrt{a} e_{1}+\sqrt{\frac{1}{2(z-a)}}\left(k_{\mathrm{I}}^{a}-\mathrm{i} k_{\mathrm{II}}^{a}\right)
\end{aligned}
$$

where

$$
\left(k_{\mathrm{I}}^{a}-\mathrm{i} k_{\mathrm{II}}^{a}\right)=\lim _{z \rightarrow a} 2(z-a)\left(t_{y y}-\mathrm{i} t_{x y}\right) \approx \lim _{z \rightarrow a}(z-a)\left[\frac{2 \chi-1}{2} \mu_{0} h^{\prime}(z) \overline{h^{\prime}(z)}\right]=\frac{1}{\mu_{0} \mu_{\mathrm{r}}^{2}} \frac{2 \chi-1}{2} a B_{0 y}^{2}
$$

and the corresponding magnetoelastic stresses of the point near the crack tip can be expressed as

$$
\begin{aligned}
t_{x x} & =\frac{k_{\mathrm{I}}}{\sqrt{2 r}} \cos \frac{\theta}{2}\left(1-\sin \frac{\theta}{2} \sin \frac{3 \theta}{2}\right)-\frac{k_{\mathrm{II}}}{\sqrt{2 r}} \sin \frac{\theta}{2}\left(2+\cos \frac{\theta}{2} \cos \frac{3 \theta}{2}\right)+\frac{k_{\mathrm{I}}^{a}}{2 r}\left(\sin ^{2} \frac{\theta}{2}+\frac{1}{2 \chi-1} \cos ^{2} \frac{\theta}{2}\right) \\
t_{y y} & =\frac{k_{\mathrm{I}}}{\sqrt{2 r}} \cos \frac{\theta}{2}\left(1+\sin \frac{\theta}{2} \sin \frac{3 \theta}{2}\right)+\frac{k_{\mathrm{II}}}{\sqrt{2 r}} \sin \frac{\theta}{2} \cos \frac{\theta}{2} \cos \frac{3 \theta}{2}+\frac{k_{\mathrm{I}}^{a}}{2 r}\left(\cos ^{2} \frac{\theta}{2}+\frac{1}{2 \chi-1} \sin ^{2} \frac{\theta}{2}\right) \\
t_{x y} & =\frac{k_{\mathrm{I}}}{\sqrt{2 r}} \sin \frac{\theta}{2} \cos \frac{\theta}{2} \cos \frac{3 \theta}{2}+\frac{k_{\mathrm{II}}}{\sqrt{2 r}} \cos \frac{\theta}{2}\left(1-\sin \frac{\theta}{2} \sin \frac{3 \theta}{2}\right)+\frac{k_{\mathrm{I}}^{a}}{2 r}\left[\frac{2(\chi-1)}{2 \chi-1} \sin \frac{\theta}{2} \cos \frac{\theta}{2}\right]
\end{aligned}
$$

Range 3: $r / a<1 /\left(50 \mu_{r}^{2}\right)$

For the present range, the terms with singularity $1 / r$ (i.e. $\mu_{0} M_{i} M_{j} / \chi$ ) are more significant, and then the stress intensity factor reduces to $k_{\mathrm{I}}^{a}-\mathrm{i} k_{\mathrm{II}}^{a}$. Then the magnetoelastic stresses of the point near the crack tip are

$$
\begin{aligned}
& t_{x x}=\frac{k_{\mathrm{I}}^{a}}{2 r}\left(\sin ^{2} \frac{\theta}{2}+\frac{1}{2 \chi-1} \cos ^{2} \frac{\theta}{2}\right), \quad t_{y y}=\frac{k_{\mathrm{I}}^{a}}{2 r}\left(\cos ^{2} \frac{\theta}{2}+\frac{1}{2 \chi-1} \sin ^{2} \frac{\theta}{2}\right), \\
& t_{x y}=\frac{k_{\mathrm{I}}^{a}}{2 r}\left[\frac{2(\chi-1)}{2 \chi-1} \sin \frac{\theta}{2} \cos \frac{\theta}{2}\right]
\end{aligned}
$$

In Eq. (80), the term $\sqrt{a} e_{1}$ induced by the boundary conditions at infinity as described previously has $1 / \sqrt{r}$ singularity near the crack tip. Alternatively, the second term $\left(k_{\mathrm{I}}^{a}-\mathrm{i} k_{\mathrm{II}}^{a}\right) /(2(z-a))^{1 / 2}$ comes from the definition of magnetoelastic stresses and has $1 / r$ singularities near the crack tip. Both terms are comparable to 
each other in the intermediate range (i.e. range 2) since $\mu_{\mathrm{r}} \gg 1$ for soft ferromagnetic materials. The contributions of these two terms to magnetoelastic stresses $t_{y y}$ and $t_{x y}$ along the real axis (i.e. $\theta=0$ ) are presented in Eq. (82) and shown in Fig. 2. The quantities $k_{\mathrm{I}} / \sqrt{2 r}, k_{\mathrm{II}} / \sqrt{2 r}$ and $k_{\mathrm{I}}^{a} / 2 r$ are generated by these two terms, respectively. Since $k_{\mathrm{II}}^{a}$ and $\theta$ are zero for the present case, the contributions of $k_{\mathrm{I}}, k_{\mathrm{I}}^{a}$ and $k_{\mathrm{II}}^{a}$ to $t_{x y}$ are absent. It is remarked by Moon (1984) that the typical magnetic induction $B_{0}=1 \mathrm{~T}$ will induce magnetic stress $B_{0}^{2} / 2 \mu_{0}=58$ psi. Such a stress will act on the soft ferromagnetic material just like that of far-field stresses $\sigma_{1}^{\infty}$ and $\sigma_{2}^{\infty}$. Since we only focus on the effect of magnetic induction, the applied stresses $\sigma_{1}^{\infty}$ and $\sigma_{2}^{\infty}$ whose effects have been studied thoroughly are dropped in all the illustrative figures. In Fig. 2, there are two dashed lines between separate range 1-3. Along the dash line between range 2 and range 3 , the

(a)

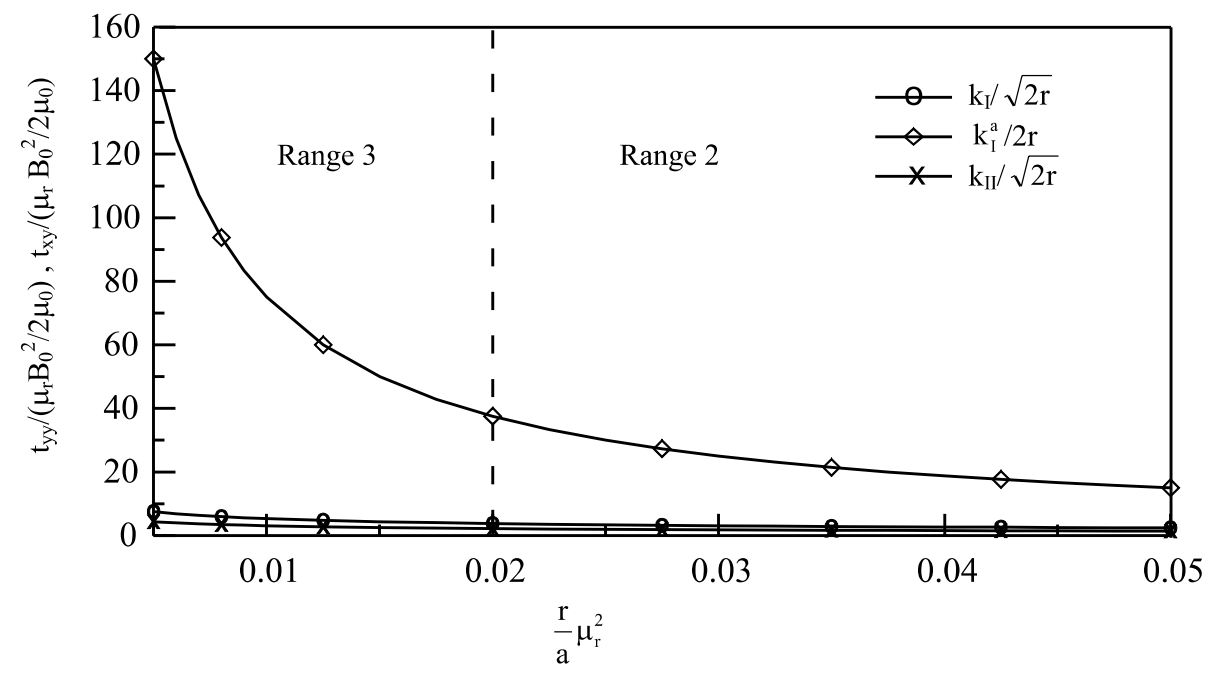

(b)

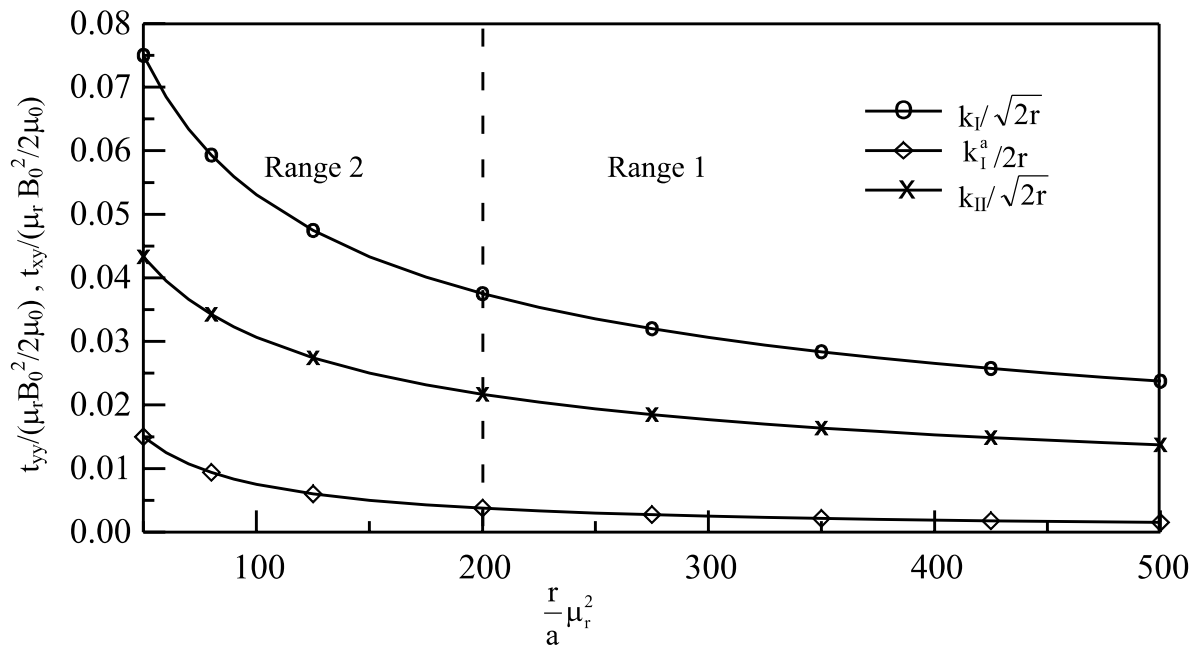

Fig. 2. The contributions of various terms on dimensionless magnetoelastic stresses along real axis with $r\left(\delta=60^{\circ}\right)$ : (a) $0.005 / \mu_{\mathrm{r}}^{2} \leqslant r / a \leqslant 0.05 / \mu_{\mathrm{r}}^{2}$ and (b) $50 / \mu_{\mathrm{r}}^{2} \leqslant r / a \leqslant 500 / \mu_{\mathrm{r}}^{2}$. 


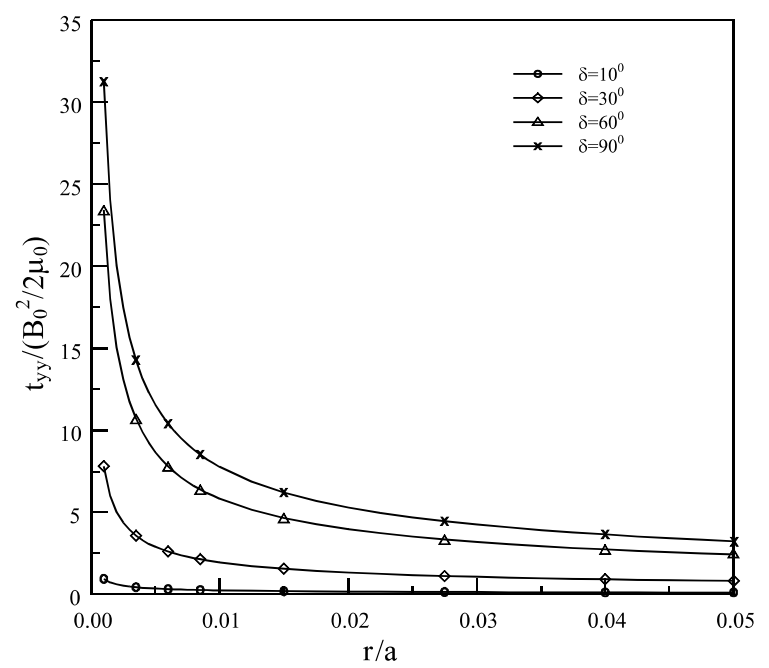

Fig. 3. The variation of dimensionless magnetoelastic stress $t_{y y} /\left(B_{0}^{2} / 2 \mu_{0}\right)$ on real axis with $r$.

magnitudes for $k_{\mathrm{I}} / \sqrt{2 r}$ and $k_{\mathrm{II}} / \sqrt{2 r}$ are one-tenth of $k_{\mathrm{I}}^{a} / 2 r$. Alternatively, the dash line between range 1 and range 2 indicates the magnitudes of $k_{\mathrm{I}}^{a} / 2 r$ is one-tenth of $k_{1} / \sqrt{2 r}$. It is guaranteed that the term with singularity $1 / r$ and the terms with singularity $1 / \sqrt{r}$ are negligible in the range 1 and the range 3 , respectively. For a typical value of $\mu_{\mathrm{r}}$ as 1000 , the intermediate range becomes $2 \times 10^{-4}>r / a \geqslant 2 \times 10^{-8}$ which is below the detectable range of instrument. Therefore, the dominant term $\sqrt{a} e_{1 \mathrm{R}}$ in range 1 is the most important. Figs. 3 and 4 display the variation of magnetoelastic stresses $t_{y y}, t_{x y}$ on positive real axis outside the crack with the distance $r$. The variations of magnetoelastic stresses to angle $\delta$ are shown in Figs. 5 and 6 , respectively. In Figs. 3-6, the value of magnetic susceptibility $\chi$ is assumed to be 100 . It is found that, when $\delta$ approaches to zero, the stresses decrease rapidly. Such a feature confirms that the component of magnetic induction parallel to the crack surface will not induce singularity.

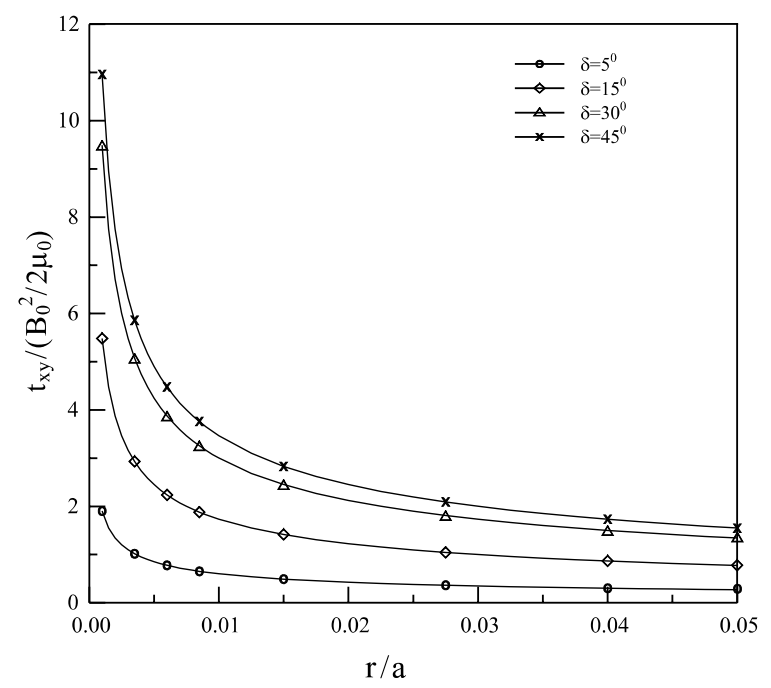

Fig. 4. The variation of dimensionless magnetoelastic stress $t_{x y} /\left(B_{0}^{2} / 2 \mu_{0}\right)$ on real axis with $r$. 


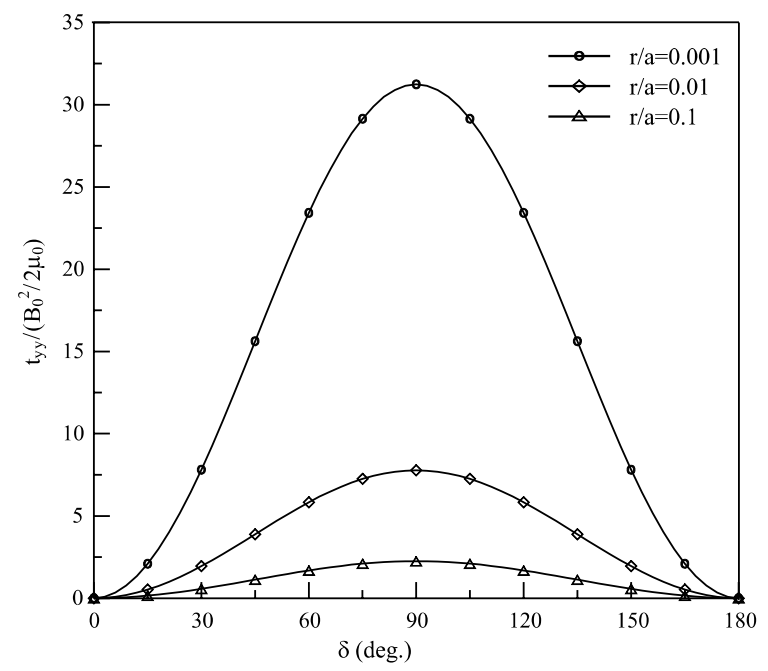

Fig. 5. The variation of dimensionless magnetoelastic stress $t_{y y} /\left(B_{0}^{2} / 2 \mu_{0}\right)$ on real axis with $\delta$.

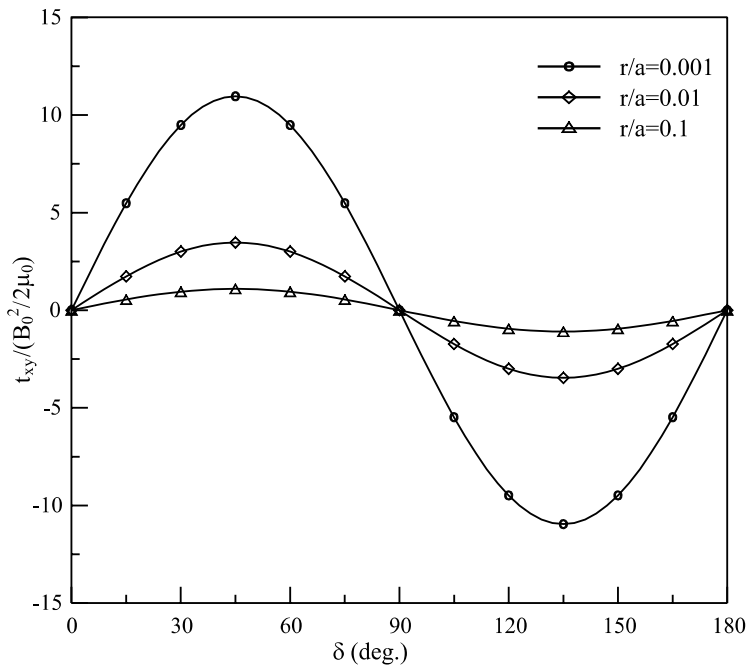

Fig. 6. The variation of dimensionless magnetoelastic stress $t_{x y} /\left(B_{0}^{2} / 2 \mu_{0}\right)$ on real axis with $\delta$.

The formulation of driving force acting on the crack within ferromagnetic materials was given for soft ferromagnet (Sabir and Maugin, 1996) and hard ferromagnets (Fomethe and Maugin, 1998) under the action of magnetic induction. For soft ferromagnets, the $J$-integral can be expressed as (Sabir and Maugin, 1996)

$$
J=\int_{\Gamma}\left[W N_{x}-n_{i} t_{i j}^{\mathrm{T}} u_{j, x}-\left(B_{k} n_{k}\right) \mu_{0} H_{x}\right] \mathrm{d} \Gamma
$$

where $W$ is magnetoelastic enthalpy and $n_{x}$ and $n_{y}$ are components of outward unit normal to the contour $\Gamma$ which is an arbitrary path surrounding the crack tip. Since the integral in Eq. (84) is path independent, it is convenient to take contour $\Gamma$ as a circle with radius $r$ and its center is located at the crack tip as shown in 
Fig. 1. The indexes $i, j$, in the equation may be space coordinates $x$ or $y$. For isotropic materials described by Eqs. (3) and (32), the magnetoelastic enthalpy becomes

$$
W=\frac{1}{2}(\lambda+2 G)\left(\varepsilon_{x x}^{2}+\varepsilon_{y y}^{2}\right)+\lambda \varepsilon_{x x} \varepsilon_{y y}+G \varepsilon_{x y}^{2}-\frac{1}{2} \mu_{0}(\chi+1)\left(H_{x}^{2}+H_{y}^{2}\right)
$$

in which $\varepsilon_{i j}$ is the strain then can be obtained from Eq. (32) as

$$
\varepsilon_{i j}=\frac{1}{2}\left(u_{i, j}+u_{j, i}\right)=\frac{\tau_{i j}}{2 G}-\frac{\lambda \delta_{i j}}{2 G} \frac{\tau_{k k}}{3 \lambda+2 G}, \quad i, j=x, y
$$

Here the relation $\tau_{z z}=v\left(\tau_{x x}+\tau_{y y}\right)$ deduced from plane strain assumption has been used. The magnetostriction terms are absent in Eq. (85) because they are much small than those terms related to magnetic susceptibility under the small strain assumption. To illustrate the effect of magnetic field on driving force $(J-$ integral) on crack, we consider the case that $\varpi$ and $B_{0 x}$ equal to zero, which leads $k_{\mathrm{II}}=0$. In order to evaluate the integral in Eq. (84), we apply the following identity

$$
\begin{aligned}
u_{x, x}+\mathrm{i} u_{y, x} & =\frac{\partial D}{\partial z}+\frac{\partial D}{\partial \bar{z}} \\
& =\frac{1}{2 G}\left\{\kappa \Phi(z)-\Omega(\bar{z})-(z-\bar{z}) \overline{\Phi^{\prime}(z)}-\frac{1}{2}\left[\frac{\chi h^{\prime}(z)}{\lambda+2 G}+\overline{h^{\prime}(z)}\right] \overline{h^{\prime}(z)}-\frac{1}{2} \frac{\mu_{0} \chi}{\lambda+2 G} \int h^{\prime}(z) \overline{h^{\prime \prime}(z)} \mathrm{d} z\right\}
\end{aligned}
$$

which can be obtained from Eqs. (42) and (49). Thereafter, substituting Eqs. (79), (85)-(87) into Eq. (84) and taking $\left(n_{x}, n_{y}\right)=(\cos \theta, \sin \theta)$, we have

$$
J=\frac{\left(1-v^{2}\right) \pi k_{\mathrm{I}}^{2}}{E}\left[1+O\left(\frac{1}{\mu_{\mathrm{r}}}\right)\right] \approx \frac{\left(1-v^{2}\right) \pi k_{\mathrm{I}}^{2}}{E}
$$

where Young's modulus $E$ and Poisson's ratio $v$ relate to $\lambda$ and $G$ by

$$
E=\frac{G(3 \lambda+2 G)}{\lambda+G}, \quad v=\frac{\lambda}{2(\lambda+G)}
$$

The result in Eq. (88) is in the same form as that given by Broek (1982) but here the $k_{\mathrm{I}}$ has an extra term which relates to the magnetic induction.

Since Eq. (59) for traction free on crack surfaces is valid only for open crack, it is necessary to check whether the crack is open or not. The derivative of relative displacement between upper and lower surfaces of crack can be derived from Eq. (69) as

$$
u_{\mathrm{r}}^{\prime}(t)=\operatorname{Im}\left[u_{x}^{\prime}(t)+\mathrm{i} u_{y}^{\prime}(t)\right]
$$

where

$$
\begin{aligned}
u_{x}^{\prime}(t)+\mathrm{i} u_{y}^{\prime}(t)= & {\left[u_{x}^{\prime}(t)+\mathrm{i} u_{y}^{\prime}(t)\right]^{+}-\left[u_{x}^{\prime}(t)+\mathrm{i} u_{y}^{\prime}(t)\right]^{-} } \\
= & \frac{1}{2 G}\left\{\left[\kappa \Phi^{+}(t)-\Omega^{-}(t)-\frac{\mu_{0}}{2} \overline{h^{\prime}}(t) \overline{h^{\prime}}(t)-\frac{G}{\lambda+2 G} \mu_{0} \chi h^{\prime^{+}}(t) \overline{h^{\prime^{+}}}(t)\right]\right. \\
& \left.\quad-\left[\kappa \Phi^{-}(t)-\Omega^{+}(t)-\frac{\mu_{0}}{2} \overline{h^{\prime+}}(t) \overline{h^{\prime^{+}}}(t)-\frac{G}{\lambda+2 G} \mu_{0} \chi h^{\prime^{-}}(t) \overline{h^{\prime-}}(t)\right]\right\}
\end{aligned}
$$

The crack opening condition

$$
\int_{-a}^{t} u_{\mathrm{r}}^{\prime}\left(t^{\prime}\right) \mathrm{d} t^{\prime} \geqslant 0 \quad \text { for } t \in L
$$

can reduce to

$$
\operatorname{Im}\left[\left.\frac{1}{2 G}(\kappa+1) e_{1} \sqrt{t^{\prime 2}-a^{2}}\right|_{-a} ^{t}\right]=\frac{1}{2 G}(\kappa+1) \sqrt{a^{2}-t^{2}}\left(e_{1 \mathrm{R}}\right) \geqslant 0
$$


The crack opening condition in Eq. (92) (i.e. $e_{1 \mathrm{R}} \geqslant 0$ ) is identical to that $k_{\mathrm{I}}$ is positive as shown in Eq. (78). The meaning of positive $k_{\mathrm{I}}$ is that the total stresses near the crack tip tend to open the crack. By observing Eqs. (78), (80), (81) and (92), the magnetic induction component $B_{0 x}$ parallel to the crack surface has no significant contribution to stress intensity factor or crack opening. But the component $B_{0 y}$ of applied magnetic induction, which is normal to the crack surface tends to open the crack since the definition of $k_{\text {I }}$ are all positive for the area outside the crack.

\section{Concluding remarks}

The solutions presented here are restricted to soft ferromagnetic material problem. A closed form solution is obtained by the application of complex variable theory. The stress intensity factors and the driving force for the crack within a soft ferromagnetic material under the action of applied magnetic field are obtained and the crack opening condition is also pointed out.

\section{Acknowledgements}

The authors wish to express their thanks to the financial support provided by National Science Council, ROC through grant no. NSC-89-2211-E-002-035.

\section{References}

Broek, D., 1982. Elementary Engineering Fracture Mechanics. Martinus Nijhoff, The Hague.

Brown Jr., W.F., 1966. Magnetoelastic Interactions. Springer, New York.

England, A.H., 1971. Complex Variable Methods in Elasticity. Wiley-Interscience, New York.

Eringen, A.C., Maugin, G.A., 1989. Electrodynamics of Continua. Springer, New York.

Fomethe, A., Maugin, G.A., 1998. On the crack mechanics of hard ferromagnets. International Journal of Non-Linear Mechanics 33, 85-95.

Maugin, G.A., 1988. Continuum Mechanics of Electromagnetic Solids. North-Holland, Amsterdam.

Moon, F.C., 1984. Magneto-solid Mechanics. Wiley, New York.

Muskhelishvili, N.I., 1953. Some Basic Problems of the Mathematical Theory of Elasticity. Noordhoff, Gronongen.

Pao, Y.H., Yeh, C.S., 1973. A linear theory for soft ferromagnetic elastic solids. International Journal of Engineering Science 11, 415436.

Sabir, M., Maugin, G.A., 1996. On the fracture of paramagnets and soft ferromagnets. International Journal of Non-Linear Mechanics 31, 425-440.

Shindo, Y., 1977. The linear magnetoelastic problem for a soft ferromagnetic elastic solid with a finite crack. ASME Journal of Applied Mechanics 44, 47-51.

Shindo, Y., 1978. Magnetoelastic interaction of a soft ferromagnetic elastic solid with a penny-shaped crack in a constant axial magnetic field. ASME Journal of Applied Mechanics 45, 291-296.

Shindo, Y., 1980. Singular stresses in a soft ferromagnetic elastic solid with two coplanar Griffith cracks. International Journal of Solids and Structures 16, 537-543.

Tiersten, H.F., 1964. Coupled magnetomechanical equations for magnetically saturated insulators. Journal of Mathematical Physics 5, $1298-1318$.

Tiersten, H.F., 1965. Variational principle for saturated magnetoelastic insulators. Journal of Mathematical Physics 6, $779-787$.

Yeh, C.S., 1989. Magnetic fields generated by a mechanical singularity in a magnetized elastic half plane. ASME Journal of Applied Mechanics 56, 89-95. 